\title{
Sarcopenia as a Novel Risk Factor for Nonalcoholic Fatty Liver Disease
}

\author{
Junghyun Noh* \\ Department of Internal Medicine, Inje University IIsan Paik Hospital, Goyang, Korea
}

Received February 24, 2020
Reviewed March 8, 2020
Accepted March 11, 2020
*Corresponding author
Junghyun Noh
(D)

https://orcid.org/0000-0002-7964-0515

Department of Internal Medicine, Inje University Ilsan Paik Hospital, 170 Juhwa-ro, Ilsanseo-gu,

Goyang 10380, Korea

Tel: $+82-31-910-6996$

Fax: +82-31-910-7518

E-mail:jhnoh@paik.ac.kr
Nonalcoholic fatty liver disease (NAFLD) is currently the most prevalent chronic liver disease worldwide, including in Korea. A recent study reported an increase in the prevalence of NAFLD from $18.6 \%$ in $1998-2001$ to $21.5 \%$ in $2016-2017$ that was accompanied by an increasing prevalence of obesity in Korea. ${ }^{1}$ NAFLD can progress to end-stage liver disease and is projected to become the main cause of liver-related mortality. Recent evidence suggested that even steatosis and mild inflammation can progress to liver cirrhosis and hepatocellular carcinoma. ${ }^{2}$ Based on these reports, the clinical significance of NAFLD has recently been highlighted. Despite the growing clinical importance of NAFLD, well-validated pharmacological treatment options are scarce. For this reason, there is a need to identify risk factors associated with the development of NAFLD to establish prevention strategies. NAFLD is thought to be the hepatic manifestation of metabolic syndrome. Obesity and insulin resistance, the main components underlying the pathogenesis of metabolic syndrome, are well-known major risk factors for NAFLD. ${ }^{3}$ Male sex, postmenopausal women, a sedentary lifestyle, age, and type 2 diabetes mellitus are also known to be important risk factors for NAFLD. ${ }^{4}$

Sarcopenia is a progressive and generalized muscle disease char- acterized by loss of muscle mass and strength. Sarcopenia is associated with an increased risk of poor health outcomes such as falls, functional decline, frailty, disability, hospitalization, and death. Sarcopenia is a commonly occurring condition in the elderly population, but it can develop in relatively young individuals due to secondary causes such as chronic organ failure, immobility, an unbalanced diet, or malignancy.

Sarcopenia has been recognized as one of the comorbidities associated with advanced liver diseases. ${ }^{5}$ Recent data have shown that obese or overweight people with chronic advanced liver diseases experience lower mortality compared with patients of normal weight, which could be attributed to less sarcopenia in patients with higher body mass index. ${ }^{6}$ Interestingly, many studies have shown that low skeletal muscle mass and muscle strength have significant associations with NAFLD, and sarcopenia has emerged as a novel risk factor for NAFLD. A recent meta-analysis of 19 studies found that the skeletal muscle index (SMI) in subjects with NAFLD was 1.77 times (95\% confidence interval [CI], 1.15-2.39) lower than that in normal controls. ${ }^{7}$ There are also several crosssectional studies that investigated the association of NAFLD and sarcopenia in the Korean population. A study from the Korean Sar- 
copenic Obesity Study showed that subjects with low muscle mass had an increased risk of NAFLD after adjusting for insulin resistance and inflammation. ${ }^{8}$ Another subsequent study showed a positive relationship between sarcopenia and NAFLD regardless of obesity or insulin resistance. ${ }^{9}$ A recent longitudinal study from a general Korean population showed that $14.8 \%$ of the 12,624 subjects without baseline NAFLD developed NAFLD during the 7-year follow-up period. Compared to the lowest SMI tertile at baseline, the highest tertile was inversely associated with incident NAFLD (adjusted hazard ratio [AHR], 0.44; 95\% CI, 0.38-0.51) and positively associated with the resolution of baseline NAFLD (AHR, 2.09; 95\% CI, 1.02-4.28). ${ }^{10}$ This study suggested that less sarcopenia over time may have beneficial effects on NAFLD development or the resolution of existing NAFLD. Sarcopenia is also known to be closely related to the severity of chronic liver disease. One study from Korea showed that the prevalence of sarcopenia increased in proportion to the severity of chronic liver disease (control: NAFLD; nonalcoholic steatohepatitis groups: 8.7\%, $17.9 \%$, and $35.0 \%$, respectively; $P<0.001) .{ }^{11}$

However, the mechanism of or related factors in the association between sarcopenia and the development of NAFLD are not completely understood. In the recent issue of Journal of Obesity \& Metabolic Syndrome, Chung et al. ${ }^{12}$ investigated the association between sarcopenia and NAFLD independent of visceral adiposity, and they searched for the clinical characteristics that affect this association. This retrospective cross-sectional study included 5,989 subjects (mean age, 53.2 years; men, 57.3\%) who underwent bioelectrical impedance analysis (BIA) and abdominal ultrasonography. In this study, the prevalence of NAFLD was significantly higher in subjects with sarcopenia than in those without $(69.5 \%$ vs. $36.5 \%$, respectively; $P<0.001)$. After adjusting for metabolic confounders (i.e., age, sex, visceral fat area, hypertension, diabetes, total and lowdensity lipoprotein cholesterol), subjects with sarcopenia showed significantly higher risk for NAFLD (odds ratio [OR], 1.37; 95\% CI, 1.02-1.84; $P=0.036)$. Subjects with sarcopenia were more likely to have a severe grade of NAFLD compared to subjects without sarcopenia (OR, 1.58; 95\% CI, 1.25-2.00; $P<0.001)$. Interestingly, there was a significant interaction in terms of effect modification in the association between sarcopenia and NAFLD by age; younger individuals showed a stronger association between sarco- penia and NAFLD $(P=0.007)$. This study reconfirmed the increased risk of NAFLD in Korean sarcopenic patients. The findings on the association between sarcopenia and NAFLD severity are consistent with previous studies. The new finding of this study is that the association between NAFLD and sarcopenia was stronger in younger subjects in the subgroup analysis. This finding may suggest that younger subjects who have sarcopenia due to secondary causes face a higher risk of NAFLD.

The limitations of this study need to be considered. Because the study design was retrospective and cross-sectional, it was not possible to evaluate the existence of a cause-effect relationship between sarcopenia and NAFLD. In addition, BIA and abdominal ultrasonography are not gold standards for the diagnosis of sarcopenia or NAFLD. Muscle strength or physical performance, which are suggested to be diagnostic criteria for sarcopenia by the European Working Group on Sarcopenia in Older People and the Asian Working Group for Sarcopenia, were not assessed in this study. Nonetheless, this study confirmed the associations between sarcopenia as well as the presence of and severity of ultrasound-graded NAFLD, independent of visceral fatness and other metabolic confounders. This study also showed that younger individuals showed a greater magnitude of association between sarcopenia and NAFLD. The possible mechanisms of this finding need to be evaluated in future prospective studies.

\section{CONFLICTS OF INTEREST}

The author declares no conflict of interest.

\section{REFERENCES}

1. Park SH, Plank LD, Suk KT, Park YE, Lee J, Choi JH, et al. Trends in the prevalence of chronic liver disease in the Korean adult population, 1998-2017. Clin Mol Hepatol 2019 Nov 4 [Epub]. https://doi.org/10.3350/cmh.2019.0065

2. McPherson S, Hardy T, Henderson E, Burt AD, Day CP, Anstee QM. Evidence of NAFLD progression from steatosis to fibrosing-steatohepatitis using paired biopsies: implications for prognosis and clinical management. J Hepatol 2015;62:114855. 
3. Marchesini G, Marzocchi R. Metabolic syndrome and NASH. Clin Liver Dis 2007;11:105-17.

4. Iqbal U, Perumpail BJ, Akhtar D, Kim D, Ahmed A. The epidemiology, risk profiling and diagnostic challenges of nonalcoholic fatty liver disease. Medicines (Basel) 2019;6:41.

5. Bhanji RA, Carey EJ, Yang L, Watt KD. The long winding road to transplant: how sarcopenia and debility impact morbidity and mortality on the waitlist. Clin Gastroenterol Hepatol 2017; 15:1492-7.

6. Karagozian R, Bhardwaj G, Wakefield DB, Baffy G. Obesity paradox in advanced liver disease: obesity is associated with lower mortality in hospitalized patients with cirrhosis. Liver Int 2016;36:1450-6.

7. Cai C, Song X, Chen Y, Chen X, Yu C. Relationship between relative skeletal muscle mass and nonalcoholic fatty liver disease: a systematic review and meta-analysis. Hepatol Int 2020; 14:115-26.

8. Hong HC, Hwang SY, Choi HY, Yoo HJ, Seo JA, Kim SG, et al. Relationship between sarcopenia and nonalcoholic fatty liver disease: the Korean Sarcopenic Obesity Study. Hepatology 2014;59:1772-8.

9. Lee YH, Jung KS, Kim SU, Yoon HJ, Yun YJ, Lee BW, et al. Sarcopaenia is associated with NAFLD independently of obesity and insulin resistance: Nationwide surveys (KNHANES 2008-2011). J Hepatol 2015;63:486-93.

10. Kim G, Lee SE, Lee YB, Jun JE, Ahn J, Bae JC, et al. Relationship between relative skeletal muscle mass and nonalcoholic fatty liver disease: a 7-year longitudinal study. Hepatology 2018; 68:1755-68.

11. Koo BK, Kim D, Joo SK, Kim JH, Chang MS, Kim BG, et al. Sarcopenia is an independent risk factor for non-alcoholic steatohepatitis and significant fibrosis. J Hepatol 2017;66:123-31.

12. Chung GE, Kim MJ, Yim JY, Kim JS, Yoon JW. Sarcopenia is significantly associated with presence and severity of nonalcoholic fatty liver disease. J Obes Metab Syndr 2019;28:129-38. 